Methods In June 2017 BAPEN sent a freedom of information (FOI) request to all trusts in England, health boards in Scotland and Wales and social care trusts in Northern Ireland. This requested information on the size of the trust, the presence and composition of the NST and their role within the hospital along with information about the trust's NSC. Those responses received before 18 th October were collated. Hospitals with fewer than 100 beds were excluded from the analysis.

Results Of the 181 FOI requests made BAPEN received responses from 122 trusts representing 154 hospitals with more than 100 beds. $91 \%$ of trusts in the UK had a NSC and only $80 \%$ of trusts reported having a NST. Of this only $25 \%$ of social care trusts in Northern Ireland and $40 \%$ of health boards in Wales reported having an NST. 48\% of trusts with an NST had a nutrition nurse, a dietician, a doctor and a pharmacist. $76 \%$ of trusts with an NST had a nutrition nurse, $79 \%$ had a pharmacist, $86 \%$ had a doctor and $88 \%$ had a dietician. $57 \%$ of NSTs performed a ward round more than once a week with $10 \%$ seeing patients as required and $4 \%$ providing an advisory role only.

Conclusion There has been a clear improvement in the provision of NSC's and NST's within the UK over time. Sadly despite this increase in NSC's and NST's we have not managed to fulfil the aim of having one in each trust. Furthermore the majority do not have the full multi-disciplinary team provision required to provide the highest level of care. More work needs to be done to promote the importance of the NSC and NST and provide support in developing them in trusts that currently do not have them ensure that all trusts have access to them.

\section{PWE-114 GASTROSTOMY INSERTION: BEYOND THE MORTALITY RATES}

${ }^{1}$ Joanne Louise Hulley*, 'Satyasheel Ramful, ${ }^{1,2}$ Nick Thompson. ${ }^{1}$ Newcastle Hospitals, Newcastle, UK; ${ }^{2}$ Northern Nutrition Network, Newcastle, UK

\subsection{6/gutjpl-2018-BSGAbstracts.348}

Introduction Short-term mortality rates from percutaneous endoscopic gastrostomy (PEG) insertion are well documented and often the focus of audit. Our Northern Nutrition Network extends across nine trusts in the North East and Cumbria, comprising of gastroenterologists, dietitians and specialist nurses. We analysed our regional population.

Methods 3 months of prospective data were collected on patients undergoing PEG or radiologically inserted gastrostomy (RIG); focussing on mortality, premorbid state, complications and sedation use. 90 day outcomes were recorded.

Results 146 gastrostomies were inserted, with a 30 day mortality rate of $8.2 \%(12 / 146)$ and 90 day mortality rate of $17.5 \%$ (26/146). Our 30 day mortality included a $2 \%$ (3/146) risk of dying in the first 7 days after gastrostomy; deemed attributable to the procedure.

Indications for gastrostomy included; ENT/UGI obstruction/ malignancy (46/146), neurological conditions (20/146), stroke (31/146), depressed consciousness (17/146), malnutrition (4/146) and failure of function (13/146). 88 patients were female, 56 were male. The average ASA was 2.7; mean BMI was 23.5; mean albumin was 37; mean age was 66 years. The 30 day mortality group had a higher mean age (76.7 vs. 66 years) and ASA (3.1 vs. 2.7$)$, and a lower BMI (18.5 vs. 23.5$)$ and albumin (31 vs. 37), suggesting these factors, which are associated with frailty, could impact on mortality.

We found a complication rate of $27 \%(40 / 146)$ and a statistically significant link between mortality and complications. The 30 day mortality increased from $4.7 \%$ (5/106) to $17.5 \%$ $(7 / 40)$ if a complication occurred $(p=0.012)$. This was reflected in the 90 day mortality group, where mortality increased from $13 \%(14 / 106)$ in the group with no complications to $30 \%(12 / 40)$ in the complication group $(p=0.018)$.

The most common complication was pneumonia, at $11 \%$ (16/147). The risk of pneumonia could be linked with sedation use, as the group receiving midazolam sedation (average $2 \mathrm{mg}$ per patient) had a pneumonia rate of $13.7 \%(15 / 109)$ whereas in the un-sedated group only one patient suffered pneumonic complications $(n=11)$. There were no reported cases of pneumonia in the 26 patients who underwent general anaesthetic for the procedure.

Conclusion We report similar mortality rates to previous studies. Our data follow the trend that older, sicker, less wellnourished patients have poorer outcomes following gastrostomy insertion than their younger, fitter, counterparts. We have shown a statistically significant link between increasing mortality and complications. Likewise, our data suggest that sedation is a risk factor for our most common complication; pneumonia. Careful patient selection and realistic conversations with patients and relatives prior to gastrostomy insertion remain paramount.

\section{PWE-115 DIETARY PATTERNS IN INFLAMMATORY BOWEL DISEASE-INTOLERANCES, QUALITY OF LIFE AND CALCIUM/VITAMIN D INTAKE}

${ }^{1}$ Rajesh Krishnamoorthy*, ${ }^{2}$ Yvonne Jeanes. ${ }^{1}$ Derby Teaching Hospitals NHS Foundation Trust, Derby, UK; ${ }^{2}$ University of Roehampton, London, UK

\subsection{6/gutjinl-2018-BSGAbstracts.349}

IntroductionFood intolerances and food avoidance are common in inflammatory bowel disease (IBD). This cross-sectional study explored the prevalence of food intolerance patterns in IBD and assessed the food related quality of life (FR-QoL) and vitamin $\mathrm{D}$ and calcium intake in IBD patients.

Methods An online questionnaire with detailed questions relating to food groups commonly avoided, food related quality of life tool and calcium and vitamin D intake was displayed on the Crohn's and Colitis UK website. Disease activity was assessed by the Minnesota IBD activity index, which is a validated, patient-defined tool that relates to the patient's perception of IBD activity over the last six months. FR-QoL was assessed by a validated questionnaire (FR-QoL-29) which comprises of 29 statements encompassing different psychosocial aspects surrounding food and eating from an IBD symptoms perspective. Details of the type of IBD, duration of disease, previous surgery and disease activity were collected. Fishers' exact test and Pearson correlation were used for statistical analysis.

Results 67 respondents (40 Crohn's, 23 ulcerative colitis, 2 unclassified and 2 microscopic colitis) participated in the survey. Food avoidance was seen in 65 (97\%) patients, with mean number of foods avoided at 6 . Vegetables were avoided in $60 \%$ of the patients, followed by wheat-based products in $56 \%$ of patients. $82 \%$ of patients reported that their IBD was active. Food related quality of life was poor in inflammatory bowel disease patients and disease activity significantly 
correlated with 16 out of the 29 statements. $60 \%$ reported that food has association with disease activity. $89 \%$ were apprehensive of eating a particular food with the fear that it might trigger their IBD symptoms. Calcium and vitamin D intake from the diet was low, with a mean of $581.8 \mathrm{mg} / \mathrm{day}$ (recommended intake $1000 \mathrm{mg} /$ day) and $282.9 \mathrm{IU} /$ day (recommended intake $400 \mathrm{IU} /$ day) respectively. $55 \%$ of patients with low calcium intake and $57 \%$ of those with a low vitamin D intake were not on supplements.

Conclusions This study highlights the high prevalence of food intolerances in the IBD community, resulting in high rate of food restrictions and less intake of foods rich in calcium and vitamin D. FR-QoL in IBD was poor. Food avoidances in IBD pose an important risk factor for poor nutrition, and majority of patients experience a low food related quality of life. Proactive assessment of food intolerances, FR-QoL and dietary intake of calcium and vitamin $\mathrm{D}$ is essential to identify and rectify underlying insufficiencies.

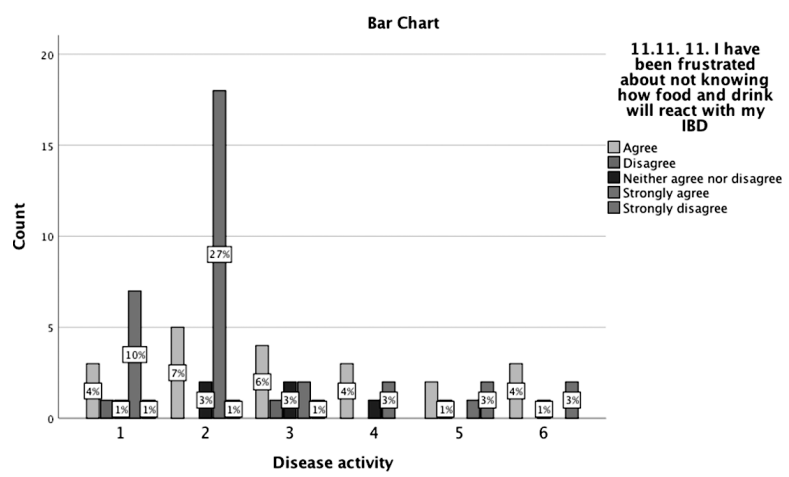

Abstract PWE-115 Figure 1 Chart showing FR-QoL statement 11 vs disease activity [Disease activity ( $\mathrm{x}$ axis): $1-4=$ active disease; $5 \& 6=$ remission $(p=0.025)$ ]

\section{REFERENCES}

1. Hughes, L. D., King, L., Morgan, M., Ayis, S., Direkze, N., Lomer, M. C., Whelan, K. (2015). Food-related quality of life in inflammatory bowel disease: development and validation of a questionnaire. Journal of Crohn's and Colitis, 10(2), 194-201.

\section{PWE-116 EVALUATING THE MANAGEMENT OF INPATIENTS WITH ANOREXIA NERVOSA: RETROSPECTIVE AUDIT USING MARSIPAN GUIDELINES}

${ }^{1}$ Buland Majeed*, ${ }^{2}$ James Hampton, ${ }^{2}$ Christian Dipper. ${ }^{1}$ Newcastle University, Newcastle upon Tyne, UK; ${ }^{2}$ Royal Victoria Infirmary, Newcastle upon Tyne, UK

\subsection{6/gutjnl-2018-BSGAbstracts.350}

Introduction The MARSIPAN guidelines were produced in response to evidence that anorexic patients on medical wards have sub-optimal outcomes. We aim to evaluate whether the care provided by a Gastroenterology ward in a busy teaching hospital meets recommendations provided by the most recent MARSIPAN guidelines.

Methods Retrospective case note analysis of patients admitted with severe anorexia nervosa $($ BMI $<15)$ over 12 months. 9 patients meeting the inclusion criteria were identified, and their care was audited against a pro-forma that was crafted according to the MARSIPAN guidelines, discussion with clinicians and the wider evidence base.

Results Our findings suggest that there is variability in compliance with the recommendations set out in the MARSIPAN guidelines. Some recommendations were met consistently; $100 \%$ of patients were seen by a dietitian and a senior psychiatrist at least once a week and had some common initial laboratory investigations (e. g. Full Blood Count, Urea and Electrolytes and Liver Function Tests). However, a number of important baseline investigations (such as the Sit-Up-SquatStand test [11.1\%], serum amylase [0\%], creatinine kinase [0\%], serum ferritin and iron [33.3\%], B12 and folate [33.3\%]) were often missed. It was also rare for a full MSE to be documented (44.4\%), or for a patient to see a senior psychiatrist twice a week or more (44.4\%). Other important risk stratifying tools such as a baseline ECG (monitoring for prolonged QT) (66.6\%) and sitting and standing blood pressure $(33.3 \%)$ were also inconsistently carried out. In regards to nursing recommendations, the majority of patients were recommended bed rest $(77.7 \%)$ and $100 \%$ had regular checks for pressure sores. However, only $22.2 \%$ were supervised for washes. $88.8 \%$ were supervised while they ate but only $22.2 \%$ were supervised for $30 \mathrm{~min}$ after (important to monitor for purging). Every patient received thiamine replacement as well as vitamin supplementation. The majority of patients were monitored daily for the first week of their admission for most markers of refeeding syndrome ( $U$ and Es [88.8\%], calcium, magnesium and phosphate [77.7\%]); however, only $33.3 \%$ had daily blood glucose measurements.

Conclusions Our findings, in the context of the wider evidence base, substantiate previous findings that anorexic patients on medical wards may receive sub-optimal care. In particular, important baseline investigations that are necessary to stratify risk are often missed and their importance needs to be better stressed. As the MARSIPAN guidelines state, 'patients near to death often look well', so being able to identify highrisk patients through rigorous investigation is key to optimising outcomes.

\section{PWE-117 VITAMIN A DEFICIENCY-NOT JUST A DEVELOPING COUNTRY PROBLEM}

Alexandra Marley*, Samuel Smith, Ruhina Ahmed, Sheldon C Cooper. Queen Elizabeth Hospital, Birmingham, UK

\subsection{6/gutjnl-2018-BSGAbstracts.351}

Introduction Vitamin A and its metabolites are required for vision, cell function for growth, reproduction, haematopoiesis, and immunity. Vitamin A deficiency is known to be associated with increased morbidity and mortality from infectious diseases. It is also known to result in visual disturbance, classically night blindness, anaemia, growth retardation and reduced fertility. Vitamin A deficiency is primary associated with the developing world, being seen in populations where malnutrition is commonplace. In 2002 it was estimated to affect 127 million preschool children and 7.2 million pregnant women worldwide. Vitamin A deficiency is being increasing seen in developed countries, invariably due to malabsorption with causes including chronic pancreatitis, chronic liver disease, intestinal failure and following bariatric surgery. Following bariatric surgery incidence of Vitamin A deficiency has been shown to be up to $69 \%$.

Our aim was to review the vitamin A deficient patients in our population, a large tertiary centre in a developed country. We wanted to review this cohort in order to obtain 